\title{
Cosmology with higher-derivative matter fields
}

\author{
Tiberiu Harko $^{a, b}$ Francisco S. N. Lobo ${ }^{c}$ Emmanuel N. Saridakis ${ }^{d, e}$ \\ ${ }^{a}$ Department of Physics, Babes-Bolyai University, Kogalniceanu Street, Cluj-Napoca 400084, Ro- \\ mania \\ ${ }^{b}$ Department of Mathematics, University College London, Gower Street, London WC1E 6BT, \\ United Kingdom \\ ${ }^{c}$ Instituto de Astrofísica e Ciências do Espaço, Faculdade de Ciências da Universidade de Lisboa, \\ Edifício C8, Campo Grande, P-1749-016 Lisbon, Portugal \\ ${ }^{d}$ Physics Division, National Technical University of Athens, 15780 Zografou Campus, Athens, \\ Greece \\ e Instituto de Física, Pontificia Universidad Católica de Valparaíso, Casilla 4950, Valparaíso, Chile \\ E-mail: t.harko@ucl.ac.uk, fslobo@fc.ul.pt, \\ Emmanuel_Saridakis@baylor.edu
}

ABSTRACT: We investigate the cosmological implications of a new class of modified gravity, where the field equations generically include higher-order derivatives of the matter fields, arising from the introduction of non-dynamical auxiliary fields in the action. Imposing a flat, homogeneous and isotropic geometry we extract the Friedmann equations, obtaining an effective dark-energy sector containing higher derivatives of the matter energy density and pressure. For the cases of dust, radiation, and stiff matter we analyze the cosmological behavior, finding accelerating, de Sitter, and non-accelerating phases, dominated by matter or dark energy. Additionally, the effective dark-energy equation-of-state parameter can be quintessence-like, cosmological-constant-like, or even phantom-like. The detailed study of these scenarios may provide signatures that could distinguish them from other candidates of modified gravity.

KeYwords: Modified gravity; Higher order derivatives; Dark energy. 


\section{Contents}

1 Introduction 1

2 Cosmology with higher-derivative matter fields $\quad 2$

2.1 Gravity with auxiliary fields 2

2.2 Cosmological equations 5

$\begin{array}{lll}3 & \text { Cosmological solutions } & 7\end{array}$

$\begin{array}{lll}3.1 & \text { Dust cosmological models } & 7\end{array}$

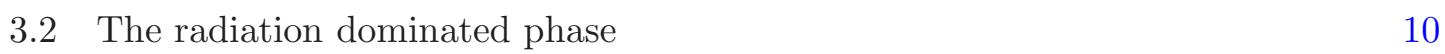

$\begin{array}{lll}3.3 & \text { Stiff fluid cosmology } & 12\end{array}$

$\begin{array}{llr}4 \text { Conclusions } & 14\end{array}$

$\begin{array}{ll}\text { A Divergence-free character of the tensor } S_{a b} & 16\end{array}$

\section{Introduction}

Since the discovery of the late-time accelerated expansion of the Universe, there has been much effort into understanding the perplexing nature of the cosmic acceleration and of gravity itself. In a first direction, one can introduce the dark-energy concept, in the context of scalar fields, as these are popular building blocks used to construct models of present-day cosmological acceleration. They are appealing because such fields are ubiquitous in theories of high energy physics beyond the standard model and, in particular, are present in theories which include extra spatial dimensions, such as those derived from string theories. Thus, the modification of the Universe content [1] is materialized, in general, by the addition of extra dynamical scalar fields, for instance canonical (quintessence) [2-7], phantom [812], their combination [13-15], K-essence [16, 17], Galileon [18], etc. On the other hand, one can modify the gravitational sector [19], for instance constructing $f(R)$ gravity [2029], $f(T)$ gravity [30-33], Weyl-Cartan-Weitzenböck gravity [34, 35], Gauss-Bonnet gravity [36-38], Hořava-Lifshitz gravity [39-43], nonlinear massive gravity [44-47], etc, which apart from the cosmological motivation has the additional advantage of an expected improved ultra-violet and quantum behavior of the theory [48]. Furthermore, one could also construct combinations of these two directions, such as in scalar-tensor theories [49-51], in generalized Galileons [52-55], etc. The common feature of all the above theories is that they incorporate additional degrees of freedom relatively to General Relativity and the standard model.

Another interesting approach is that one could handle the gravitational and matter sectors "democratically", that is modifying the matter part in the Lagrangian too, along with its coupling to gravity [56-68]. Generally, these theories lead to non-geodesic motion, 
which takes place in the presence of an extra force orthogonal to the four-velocity. The Newtonian limit of the equation of motion was also considered, and a procedure for obtaining the energy-momentum tensor of the matter was presented. On the other hand, the gravitational field equations are equivalent to the Einstein equations of the $f(R)$ model in empty spacetime, but differ from them, as well as from standard General Relativity, in the presence of matter. Therefore, the predictions of these models could lead to some major differences, as compared to the predictions of standard General Relativity, or its extensions ignoring the role of matter, in several problems of current interest, such as cosmology, gravitational collapse or the generation of gravitational waves. The study of these phenomena may also provide some specific signatures and effects, which could distinguish and discriminate between the various theories of modified gravity.

Having these in mind, one could try to construct theories with additional auxiliary fields which can alter the dynamics, however being themselves non-dynamical and thus without altering the degrees of freedom [69-71] (for similar constructions see [72-74]). Interestingly enough, the requirements of satisfying the weak equivalence principle and allowing for a covariant Lagrangian formulation, lead the field equations of these theories to include, in general, higher-order derivatives of the matter fields. Although this feature places tight observational constraints on these theories, it is clear that they correspond to novel modified classes.

The plan of the work is the following: In Section 2, we present the gravitational modification with non-dynamical auxiliary fields, extracting the corresponding cosmological equations. In Section 3, we solve the cosmological field equations and we provide the cosmological behavior for various matter equations of state. Finally, in Section 4 we briefly discuss and conclude our paper.

\section{Cosmology with higher-derivative matter fields}

In this section we extract the cosmological equations of the scenario at hand. In a first subsection, we briefly present the underlying gravitational theory, namely gravity with auxiliary fields. Then, we apply it in a cosmological framework, providing the Friedmann equations and defining the basic observables.

\subsection{Gravity with auxiliary fields}

The key point of the scenario at hand is that one introduces auxiliary fields whose equations of motion are not dynamical [69]. Thus, one can use these equations to eliminate the auxiliary fields. In this way, the equations of motion for the usual matter fields are not modified (which is a significant advantage when it comes to the observational tests of the theory), however the gravitational equations of motion do change, and in particular they acquire higher order derivatives of the matter fields. More specifically, the field equations can be written, without loss of generality, as $G_{a b}+\Lambda g_{a b}=T_{a b}+S_{a b}(\mathbf{g}, \mathbf{T})$, where the second rank tensor $S_{a b}(\mathbf{g}, \mathbf{T})$ depends explicitly on the metric $\mathbf{g}$ and the matter fields ( $\mathbf{T}$ in general). The tensor $S_{a b}$ is imposed to vanish in vacuum and be divergence-free, in virtue of the Bianchi identity and the conservation of the matter fields, namely $\nabla_{a} T^{a b}=0$. 
Thus, in the present context, these theories with auxiliary fields consist in modifying the Einstein field equation through the addition of a divergence-free tensor, that vanishes in the vacuum, and depends on the metric, the matter energy-momentum tensor, and its derivatives.

The above conditions place strict requirements on the second-rank tensor under consideration. More specifically, the tensor $S_{a b}$, up to fourth order in the derivatives, is given by (we refer the reader to Ref. [69] for more details)

$$
\begin{aligned}
S_{a b}= & \alpha_{1} g_{a b} T+\alpha_{2} g_{a b} T^{2}+\alpha_{3} T T_{a b}+\alpha_{4} g_{a b} T_{c d} T^{c d}+\alpha_{5} T^{c}{ }_{a} T_{c b} \\
& +\beta_{1} \nabla_{a} \nabla_{b} T+\beta_{2} g_{a b} \square T+\beta_{3} \square T_{a b}+2 \beta_{4} \nabla^{c} \nabla_{(a} T_{b) c}+\ldots
\end{aligned}
$$

Thus, in order to obtain $\nabla_{a} S^{a b}=0$, to the considered order of expansion, one needs to impose the conditions

$$
\begin{array}{r}
\alpha_{1}=-\beta_{1} \Lambda, \quad 4 \alpha_{2}=\left(1+2 \alpha_{1}\right)\left(\beta_{1}-\beta_{4}\right), \quad \alpha_{3}=\beta_{4}\left(1+2 \alpha_{1}\right)-\beta_{1}, \\
2 \alpha_{4}=\beta_{4}, \quad \alpha_{5}=-2 \beta_{4}, \quad \beta_{2}=-\beta_{1}, \quad \beta_{3}=-\beta_{4} .
\end{array}
$$

We refer the reader to Appendix (A) for specific details.

Hence, independently of the specific initial action, the resulting Einstein equations become [69]

$$
\begin{aligned}
G_{a b}= & T_{a b}-\Lambda g_{a b}-\beta_{1} \Lambda g_{a b} T+\frac{1}{4}\left(1-2 \beta_{1} \Lambda\right)\left(\beta_{1}-\beta_{4}\right) g_{a b} T^{2} \\
& +\left[\beta_{4}\left(1-2 \beta_{1} \Lambda\right)-\beta_{1}\right] T T_{a b}+\frac{1}{2} \beta_{4} g_{a b} T_{c d} T^{c d}-2 \beta_{4} T^{c}{ }_{a} T_{c b} \\
& +\beta_{1} \nabla_{a} \nabla_{b} T-\beta_{1} g_{a b} \square T-\beta_{4} \square T_{a b}+2 \beta_{4} \nabla^{c} \nabla_{(a} T_{b) c}+\ldots,
\end{aligned}
$$

where $g_{a b}$ is the spacetime metric, $\Lambda$ the cosmological constant, $T_{a b}$ the matter energymomentum tensor, $T$ its trace, and we have set Newton's constant to $1 / 8 \pi$ (we refer the reader to [69] for more details). The divergence of the extra terms is zero, and the coefficients now only depend on the $\beta_{1}$ and $\beta_{4}$ model parameters, and it is clear that when $\beta_{1}=\beta_{4}=0$ we recover the standard Einstein equations. In the above equation one can clearly see that the introduction of auxiliary fields indeed leads to gravitational equations which include higher derivatives of the energy momentum tensor and its trace.

Before proceeding to the cosmological investigation of the above scenario, let us make a comment on the subtle issue of considering the trace of the energy-momentum tensor, $T$, in the Lagrangian. Indeed, note that the most obvious generalization of Newtonian gravity, based on Poisson's equation $\nabla^{2} \phi=4 \pi G \rho$ should be $\square \phi=4 \pi G T$, where the trace of the energy-momentum tensor acts as a source for the scalar field. This has several implications, such as: (i) the theory is nonlinear as the scalar field possesses a non-zero trace for the energy-momentum tensor, which appears in the right-hand-side of the above relationship; (ii) the scalar field also couples to the cosmological constant, as the latter has a non-zero energy-momentum trace, thus rendering a dynamical effective cosmological constant. In this context, the subtle issue is that the trace of the energy-momentum tensor should not be written a priori inside the Lagrangian but should be derived by varying the Lagrangian. 
Thus, the correct coupling would be the one with the properly defined trace, leading then to an infinite loop of variations [75-78]. To this effect, consider a system consisting of gravitational fields $g_{\mu \nu}$, radiation fields, and a scalar field $\phi$ which couples to the trace of the energy-momentum tensor of all the fields, including its own [75-80]. Furthermore, consider the zeroth order action given by

$$
A^{(0)}=A_{\text {grav }}+A_{\phi}^{(0)}+A_{\text {int }}^{(0)}+A_{\text {rad }} .
$$

The respective terms are defined in the following manner

$$
\begin{aligned}
A_{\text {grav }} & =\frac{1}{16 \pi G} \int d^{4} x \sqrt{-g} R+\int d^{4} x \sqrt{-g} \Lambda, \\
A^{(0)} & =\frac{1}{2} \int d^{4} x \sqrt{-g} \nabla^{\mu} \phi \nabla_{\mu} \phi, \\
A_{\text {int }}^{(0)} & =\int d^{4} x \sqrt{-g} f\left(\phi / \phi_{0}\right) T,
\end{aligned}
$$

where the cosmological constant is included. $\eta$ determines the strength of the interaction between the scalar field and the trace of the energy-momentum tensor, the constant $\phi_{0}$ is introduced for dimensional convenience, and finally note that the radiation term is traceless. Now, in order to take into account the back-reaction of the scalar field on itself, the trace of the scalar field, i.e. $T_{\phi}=-\nabla^{\mu} \phi \nabla_{\mu} \phi$, must be added to the total trace $T$. Next, the addition of $T_{\phi}$ in the interaction term $A_{\text {int }}^{(0)}$ further modifies the energy-momentum tensor $T_{\phi}^{\mu \nu}$, and consequently modifies the trace $T_{\phi}$. In conclusion, an infinite iteration needs to be performed to arrive at the correct action, where the complete action is obtained by summing up all the terms [75-78].

Nevertheless, one should emphasize that the full action can be deduced by a simple consistency argument $[79,80]$. As the above-mentioned iteration is to modify the expressions for $A_{\phi}$ and $A_{\Lambda}$, one could consider the following as an ansatz for the full action

$$
\begin{array}{r}
A=\frac{1}{16 \pi G} \int d^{4} x \sqrt{-g} R+\int d^{4} x \sqrt{-g} \alpha(\phi) \Lambda \\
+\frac{1}{2} \int d^{4} x \sqrt{-g} \beta(\phi) \nabla^{\mu} \phi \nabla_{\mu} \phi+A_{\mathrm{rad}},
\end{array}
$$

where the functions $\alpha(\phi)$ and $\beta(\phi)$ represent the effect of the iteration of the interaction term. Note that taking into account the action (2.8), the energy-momentum tensor for the scalar field $\phi$ and the cosmological constant $\Lambda$ is now given by $T^{\mu \nu}=\alpha(\phi) \Lambda g^{\mu \nu}+$ $\beta(\phi)\left(\nabla^{\mu} \phi \nabla^{\nu} \phi-\frac{1}{2} g^{\mu \nu} \nabla^{\alpha} \phi \nabla_{\alpha} \phi\right)$, which provides the following trace: $T_{\text {tot }}=4 \alpha(\phi) \Lambda-$ $\beta(\phi) \nabla^{\alpha} \phi \nabla_{\alpha} \phi$. Using the latter trace equation, and equating the actions (2.4) and (2.8), the functions $\alpha(\phi)$ and $\beta(\phi)$ are given by

$$
\alpha(\phi)=(1+4 \eta f)^{-1}, \quad \beta(\phi)=(1+2 \eta f)^{-1},
$$

respectively. The cosmological behaviour was further extensively explored [79, 80]. Now, this model is conceptually attractive as it correctly accounts for the coupling of the scalar field and the trace of the energy-momentum tensor. However, there are several problems 
[79], namely: (i) one does not arrive at a viable model using natural initial conditions without fine-tuning the parameters; (ii) due to the coupling of the scalar field to the trace of all the sources, it also couples and consequently kills off the dust-like matter, rendering the present universe radiation-dominated; (iii) the latter point reduces the age of the universe and creates difficulties for the formation of structure. It has been emphasized that these problems may be solved by invoking a suitable potential $V(\phi)$, but this eliminates the naturalness of the model $[79,80]$.

In the present context, one may also argue that the theory analyzed in this work suffers form similar drawbacks in that one should not place the trace of the energy-momentum tensor inside the Lagrangian, but he should derive the latter by variation. Thus, the correct coupling would be the one with the properly defined trace, leading then to an infinite loop of variations. However, we emphasize that we may consider the theory at a phenomenological level, where we have postulated the trace in the Lagrangian, and extensively explore its consequences and cosmological behavior. This is what we do in the rest of the manuscript.

\subsection{Cosmological equations}

In order to apply the above modified gravitational theory in cosmology we consider the usual homogeneous and isotropic geometry, given by the flat Friedmann-Robertson-Walker (FRW) metric

$$
d s^{2}=-d t^{2}+a^{2}(t) \delta_{i j} d x^{i} d x^{j},
$$

where $a(t)$ is the scale factor. Additionally, concerning the matter energy-momentum tensor, we use the standard form of a perfect fluid, namely $T_{a b}=(\rho+p) U_{a} U_{b}+p g_{a b}$, where $\rho$ and $p$ are respectively the matter energy density and pressure.

Inserting these in the field equations (2.3), we obtain the modified Friedmann equations as

$$
\begin{aligned}
3 H^{2}= & (\rho+\Lambda)+3 H\left[\beta_{1}(\dot{\rho}-3 \dot{p})-\beta_{4}(\dot{\rho}+2 \dot{p})\right]-\beta_{4} \ddot{\rho} \\
& +\frac{1}{4}\left[3 \beta_{1}\left(1+2 \beta_{4} \Lambda\right)\left(\rho^{2}-3 p^{2}\right)+3 \beta_{4}\left(\rho^{2}+p^{2}\right)-12 \beta_{1}\left(\beta_{1}+\beta_{4}\right) \Lambda \rho p\right. \\
& \left.-6\left(\beta_{1}-\beta_{4}\right) \rho p-4 \beta_{1} \Lambda(\rho-3 p)+2 \beta_{1}^{2} \Lambda\left(\rho^{2}+9 p^{2}\right)\right],
\end{aligned}
$$

and

$$
\begin{aligned}
\dot{H}\left[1+\beta_{4}(\rho+p)\right]= & -\frac{1}{2}(\rho+p)-3 H^{2} \beta_{4}(\rho+p)-\frac{H}{2}\left[\beta_{1}(\dot{\rho}-3 \dot{p})-\beta_{4}(\dot{\rho}+3 \dot{p})\right] \\
& -\frac{1}{2}\left[\left(3 \beta_{1}+\beta_{4}\right) \ddot{p}-\left(\beta_{1}+\beta_{4}\right) \ddot{\rho}+\beta_{4}\left(\rho^{2}+p^{2}\right)\right. \\
& \left.+\beta_{1}\left(1+2 \beta_{4} \Lambda\right)\left(\rho^{2}-3 p^{2}\right)-2\left(\beta_{1}-\beta_{4}+2 \beta_{1} \beta_{4} \Lambda\right) \rho p\right],
\end{aligned}
$$

respectively. The above equations can be re-written in the form of the usual Friedmann equations as

$$
\begin{gathered}
3 H^{2}=\rho+\rho_{D E}, \\
-2 \dot{H}=\rho+\rho_{D E}+p+p_{D E},
\end{gathered}
$$


where we have introduced the effective dark-energy sector with energy density and pressure, $\rho_{D E}$ and $p_{D E}$, respectively, defined as

$$
\begin{aligned}
\rho_{D E}= & \Lambda+\frac{1}{4}\left[3 \beta_{1}\left(1+2 \beta_{4} \Lambda\right)\left(\rho^{2}-3 p^{2}\right)+3 \beta_{4}\left(\rho^{2}+p^{2}\right)-12 \beta_{1}\left(\beta_{1}+\beta_{4}\right) \Lambda \rho p-6\left(\beta_{1}-\beta_{4}\right) \rho p\right. \\
& \left.-4 \beta_{1} \Lambda(\rho-3 p)+2 \beta_{1}^{2} \Lambda\left(\rho^{2}+9 p^{2}\right)\right]+3 H\left[\beta_{1}(\dot{\rho}-3 \dot{p})-\beta_{4}(\dot{\rho}+2 \dot{p})\right]-\beta_{4} \ddot{\rho},
\end{aligned}
$$

and

$$
\begin{aligned}
p_{D E}= & \frac{1}{4\left[\beta_{4}(p+\rho)+1\right]}\left\{-\beta_{4} \rho^{3}\left[2 \beta_{1}\left(\beta_{1}+3 \beta_{4}\right) \Lambda+3\left(\beta_{1}+\beta_{4}\right)\right]-\left(\beta_{1}-3 \beta_{4}\right) \rho^{2}\left(2 \beta_{1} \Lambda-1\right)\right. \\
& +4 \rho\left(\beta_{1} \Lambda-\beta_{4} \Lambda+6 \beta_{4} H^{2}\right)+4 p\left[6 \beta_{4} H^{2}-\left(3 \beta_{1}+\beta_{4}\right) \Lambda\right] \\
& +\beta_{4} p \rho^{2}\left[\beta_{1}\left(10 \beta_{1} \Lambda+6 \beta_{4} \Lambda+3\right)-9 \beta_{4}\right]+2 p \rho\left[6 \beta_{1}\left(\beta_{1}-\beta_{4}\right) \Lambda-\left(\beta_{1}+3 \beta_{4}\right)\right] \\
& +3 p^{2}\left[\beta_{4} \rho\left(-2\left(\beta_{1}-5 \beta_{4}\right) \beta_{1} \Lambda+5 \beta_{1}-3 \beta_{4}\right)-\left(\beta_{1}+\beta_{4}\right)\left(6 \beta_{1} \Lambda+1\right)\right] \\
& -4 \Lambda+3 \beta_{4} p^{3}\left[6\left(\beta_{4}-\beta_{1}\right) \beta_{1} \Lambda+3 \beta_{1}-\beta_{4}\right] \\
& \left.+4 H\left[\left(\beta_{1}-\beta_{4}\right) \dot{\rho}(t)-3\left(\beta_{1}+\beta_{4}\right) \dot{p}(t)\right]+4\left(3 \beta_{1}+\beta_{4}\right) \ddot{p}-4\left(\beta_{1}+\beta_{4}\right) \ddot{\rho}\right\} .
\end{aligned}
$$

Thus, we can calculate the equation-of-state parameter $w_{D E}$ of the effective dark-energy sector as $w_{D E} \equiv p_{D E} / \rho_{D E}$, or using the Friedmann equations (2.13) and (2.14) as

$$
w_{D E}=-\frac{2 \dot{H}+p+3 H^{2}}{3 H^{2}-\rho} .
$$

Moreover, one can see that given the matter energy conservation

$$
\dot{\rho}+3 H(\rho+p)=0
$$

the effective dark energy is also conserved, that is

$$
\dot{\rho}_{D E}+3 H\left(\rho_{D E}+p_{D E}\right)=0
$$

Finally, we can define the deceleration parameter $q$, namely

$$
q=-1-\frac{\dot{H}}{H^{2}}=\frac{1}{2}+\frac{3}{2} \frac{p+p_{D E}}{\rho+\rho_{D E}} .
$$

The sign of $q$ indicates the decelerating/accelerating nature of the cosmological expansion; cosmological models with $q<0$ are accelerating, while those having $q>0$ experience a decelerating evolution. Note that in terms of $q$ the dark-energy equation-of-state parameter reads

$$
w_{D E}=\frac{(2 q-1) H^{2}-p}{3 H^{2}-\rho}
$$

or, alternatively,

$$
w_{D E}=\frac{1}{3}(2 q-1)\left(\frac{\rho}{\rho_{D E}}+1\right)-\frac{p}{\rho_{D E}} .
$$


In order to be able to handle the cosmological equations of the scenario at hand, namely the Friedmann equations (2.13) and (2.14) and the conservation equations (2.18) (or (2.19)), we proceed as follows. Firstly, from equation (2.18) we easily obtain

$$
H=-\frac{\dot{\rho}}{3(\rho+p)}
$$

and

$$
\dot{H}=\frac{\dot{\rho}(\dot{p}+\dot{\rho})-(p+\rho) \ddot{\rho}}{3(p+\rho)^{2}} .
$$

Then, substitution into equations (2.11) and (2.12) provides the equations

$$
\begin{aligned}
\beta_{4} \ddot{\rho}= & \Lambda+\rho+\frac{\dot{\rho}\left[\left(3 \beta_{1}+2 \beta_{4}\right) \dot{p}+\left(\beta_{4}-\beta_{1}\right) \dot{\rho}\right]}{p+\rho}-\frac{\dot{\rho}^{2}}{3(p+\rho)^{2}} \\
+ & \frac{1}{4}\left\{-2 \rho\left\{2 \beta_{1} \Lambda+3 p\left[2 \beta_{1} \Lambda\left(\beta_{1}+\beta_{4}\right)+\beta_{1}-\beta_{4}\right]\right\}+\rho^{2}\left[2 \beta_{1} \Lambda\left(\beta_{1}+3 \beta_{4}\right)+3\left(\beta_{1}+\beta_{4}\right)\right]\right. \\
& \left.+3 p\left[4 \beta_{1} \Lambda+p\left(6 \beta_{1} \Lambda\left(\beta_{1}-\beta_{4}\right)-3 \beta_{1}+\beta_{4}\right)\right]\right\}
\end{aligned}
$$

and

$$
\begin{gathered}
\ddot{\rho}=-\left(3 \beta_{1}+5 \beta_{4}+\frac{2}{p+\rho}\right)^{-1}\left\{3 p\left[2 \rho\left(2 \beta_{1} \beta_{4} \Lambda+\beta_{1}-\beta_{4}\right)-1\right]+3 p^{2}\left[\beta_{1}\left(6 \beta_{4} \Lambda+3\right)-\beta_{4}\right]\right. \\
-\frac{3\left(3 \beta_{1}+\beta_{4}\right)(p+\rho)^{2} \ddot{p}+\dot{p} \dot{\rho}\left[\left(3 \beta_{1}+5 \beta_{4}\right)(p+\rho)+2\right]-\dot{\rho}^{2}\left[\left(\beta_{1}-5 \beta_{4}\right)(p+\rho)-2\right]}{(p+\rho)^{2}} \\
\left.-3 \rho^{2}\left(2 \beta_{1} \beta_{4} \Lambda+\beta_{1}+\beta_{4}\right)-3 \rho\right\},
\end{gathered}
$$

Finally, eliminating $\ddot{\rho}$ between equations (2.25) and (2.26), through equalizing the right hand sides, we obtain a differential equation for $\rho$. Hence, if we additionally provide the matter equation of state, assumed to be of barotropic form $p=p(\rho)$, this differential equation can be solved to give $\rho(t)$. Lastly, $H(t)$ is then obtained from equation (2.23) and then $\rho_{D E}(t)$ from equation (2.15), $w_{D E}$ from equation (2.17) and $q$ from equation (2.20).

\section{Cosmological solutions}

In this section we explore the cosmological scenario with higher-matter derivatives, for three particular cases of matter equations of state, namely dust $(p=0)$, radiation $(p=\rho / 3)$ and stiff fluid $(p=\rho)$.

\subsection{Dust cosmological models}

As a first example we consider that the matter is in the form of dust, namely with $p=0$. In this case equations (2.25) and (2.26) are respectively simplified to

$$
\ddot{\rho}=\frac{3 \rho^{2}\left\{4 \Lambda+\rho\left\{4-4 \beta_{1} \Lambda+\rho\left[2 \beta_{1} \Lambda\left(\beta_{1}+3 \beta_{4}\right)+3\left(\beta_{1}+\beta_{4}\right)\right]\right\}\right\}-4 \dot{\rho}^{2}\left[3\left(\beta_{1}-\beta_{4}\right) \rho+1\right]}{12 \beta_{4} \rho^{2}},
$$


and

$$
\ddot{\rho}=\frac{3 \rho^{3}\left[\rho\left(2 \beta_{1} \beta_{4} \Lambda+\beta_{1}+\beta_{4}\right)+1\right]+\dot{\rho}^{2}\left[2-\left(\beta_{1}-5 \beta_{4}\right) \rho\right]}{\rho\left[\left(3 \beta_{1}+5 \beta_{4}\right) \rho+2\right]} .
$$

Hence, as we mentioned in the previous section, equality of the right-hand-sides provides the differential equation for $\rho(t)$. If for convenience we introduce the dimensionless variables

$$
\tau=\frac{t}{2 \beta_{4}^{1 / 2}}, \quad \theta=\beta_{4} \rho, \quad k=\frac{\beta_{1}}{\beta_{4}}, \quad h=\beta_{4}^{1 / 2} H, \quad \lambda=\beta_{4} \Lambda,
$$

the differential equation takes the following form

$$
\begin{aligned}
\frac{d \theta}{d \tau}= & -\theta\{\theta[3 k(\theta+3 \theta k+3)+5]+2\}^{-1 / 2}\{3 \theta(\theta+3 \theta k+4)[3 \theta(k+1)+2] \\
& \left.+6 \lambda\left\{\theta\left\{k\left[\theta^{2}\left(3 k^{2}+14 k+3\right)-4 \theta(k+1)+2\right]+10\right\}+4\right\}\right\}^{1 / 2},
\end{aligned}
$$

where for physical reasons (monotonically decreasing energy density) we have adopted the minus sign for the square root. Additionally, calculating $H(t)$ from equation (2.23) and inserting into equation (2.20) we extract the analytical expression for the deceleration parameter as

$$
\begin{gathered}
q=\left\{[ 6 k \theta ( \theta + 3 k \theta + 3 ) + 4 ] \left\{\theta \left[\theta^{2} k^{2}(6 k \lambda+28 \lambda+9)+\theta^{2}(6 k \lambda+12 k+3)\right.\right.\right. \\
\left.\left.\left.+2 \theta\left(9 k+7-4 \lambda k^{2}-\lambda k\right)+4 \lambda(k+5)+8\right]+8 \lambda\right\}\right\}^{-1} \times \\
\left\{3 \theta^{5}\left(3 k^{2}+k\right)\left[k^{2}(6 k \lambda+28 \lambda+9)+6 k(\lambda+2)+3\right]-\theta^{2}[8 k(89 k+77) \lambda-112]\right. \\
-\theta^{3}\left[8 k^{2} \lambda(21 k+80)+72 k^{2}+2 k(108 \lambda-87)-42\right] \\
\left.+3 \theta^{4}\left[8 k\left(15 \lambda k^{3}+50 \lambda k^{2}+11 \lambda k\right)+8 k(3 k+1)\right]+8 \theta \lambda(5-44 k)+16 \theta-32 \lambda\right\} .
\end{gathered}
$$

Note that for the specific case where the cosmological constant is absent (namely $\lambda=0$ ), that is when the effective dark energy sector constitutes solely from the higher matter derivatives, we obtain

$$
q=\frac{1}{2}+\frac{3(9 k \theta+4)}{6 k \theta(\theta+3 k \theta+3)+4}-\frac{6}{\theta+3 k \theta+4}-\frac{3}{3(k+1) \theta+2} .
$$

In order to present the above behavior more transparently, we numerically solve equation (3.4), and then we calculate the various physical quantities as we described above. In Figs. 1-5 we present the time evolution of the matter energy density, of the scale factor, of the Hubble function, of the deceleration parameter and of the dark-energy equation-of-state parameter, for the cases $\lambda=0$ and $\lambda=0.004$, and for different values of $k$. 

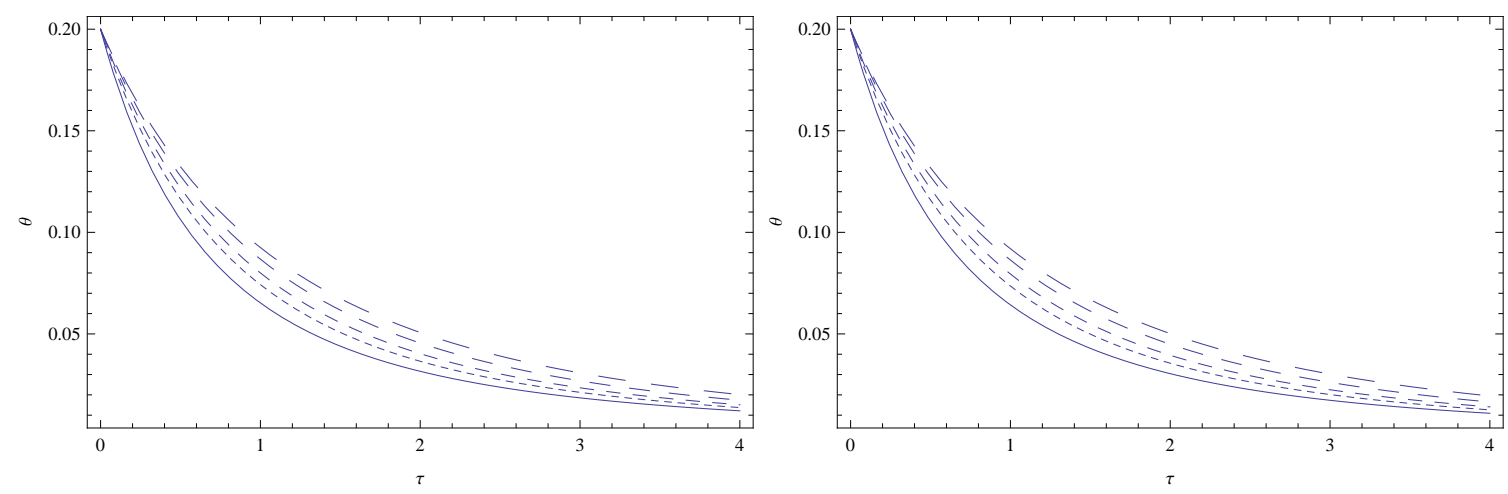

Figure 1. Time evolution of the scaled matter energy density $\theta$ of the dust-matter Universe, as a function of $\tau$, for $\lambda=0$ (left panel) and for $\lambda=0.004$ (right panel), for different values of $k: k=1$ (solid curve), $k=3$ (dotted curve), $k=5$ (short dashed curve), $k=9$ (dashed curve) and $k=16$ (long dashed curve). The initial value of the energy density is $\theta(0)=0.20$.
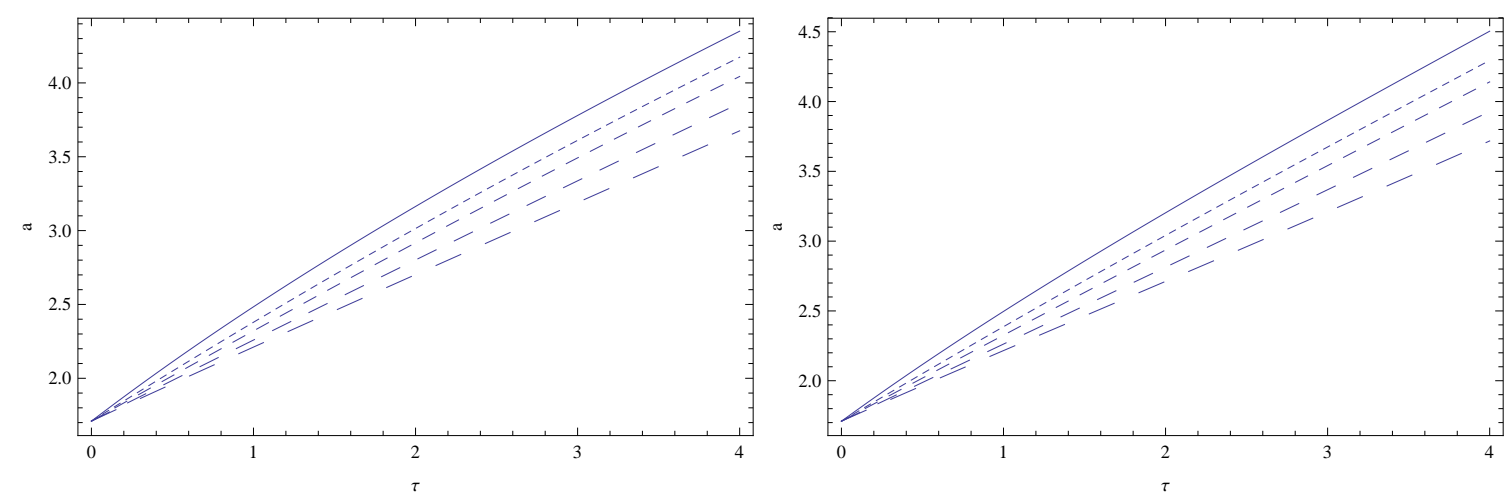

Figure 2. Time evolution of the scale factor a of the dust-matter Universe, as a function of $\tau$, for $\lambda=0$ (left panel) and for $\lambda=0.004$ (right panel), for different values of $k: k=1$ (solid curve), $k=3$ (dotted curve), $k=5$ (short dashed curve), $k=9$ (dashed curve) and $k=16$ (long dashed curve). The initial value of the energy density is $\theta(0)=0.20$.

First of all, as expected, the cosmological dynamics depends on the value of $\lambda$. In particular, since in the scenario at hand the effective dark energy sector is attributed to the higher derivatives of the matter fields, when an explicit cosmological constant is absent $(\lambda=0)$ we expect the dark-energy equation-of-state parameter to become asymptotically zero at late times, and the Universe to be non-accelerating. This behavior can be clearly seen in the left graphs of Figs. 4 and 5. On the other hand, when $\lambda \neq 0$, the explicit cosmological constant dominates inside the effective dark energy sector, and in this case the quintessence-like Universe at late times transits to the accelerating phase, tending asymptotically to the de Sitter evolution, with $w_{D E} \rightarrow-1$. This behavior can be observed in the right graphs of Figs. 4 and 5.

The above asymptotic behaviors can be also analytically extracted from the cosmological equations. In particular, setting $\lambda=0$ in (3.6) leads immediately to $\lim _{\theta \rightarrow 0} q=1 / 2$. On the other hand, for $\lambda>0$, expression (3.5) leads to $\lim _{\theta \rightarrow 0} q=-1$. 

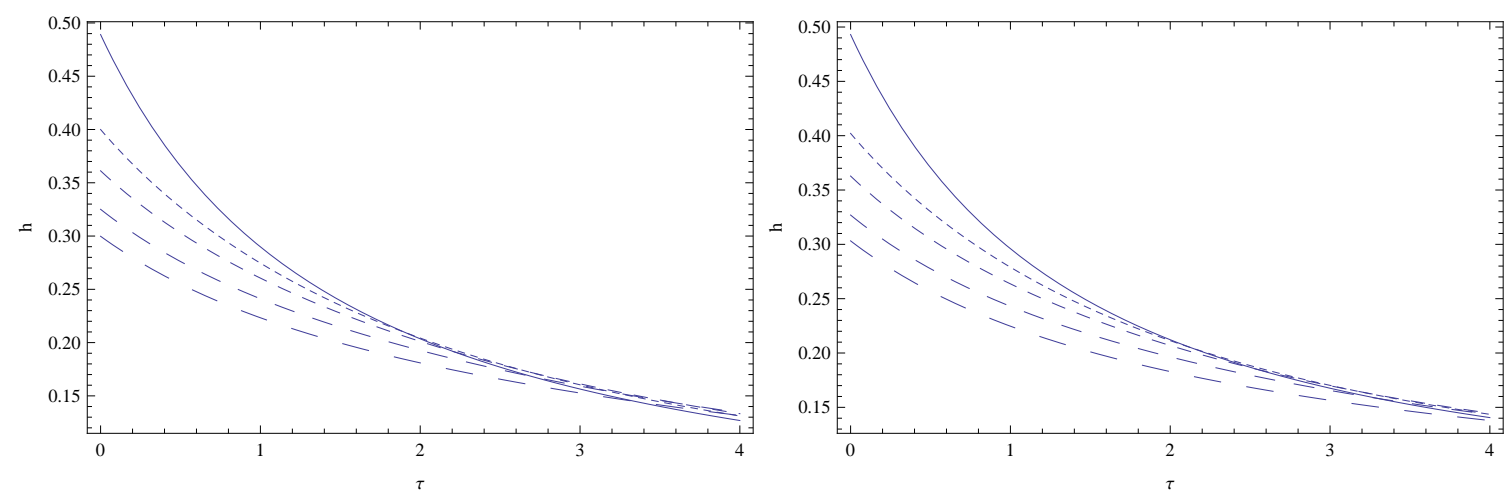

Figure 3. Time evolution of the Hubble function $h$ of the dust-matter Universe, as a function of $\tau$, for $\lambda=0$ (left panel) and for $\lambda=0.004$ (right panel), for different values of $k: k=1$ (solid curve), $k=3$ (dotted curve), $k=5$ (short dashed curve), $k=9$ (dashed curve) and $k=16$ (long dashed curve). The initial value of the energy density is $\theta(0)=0.20$.
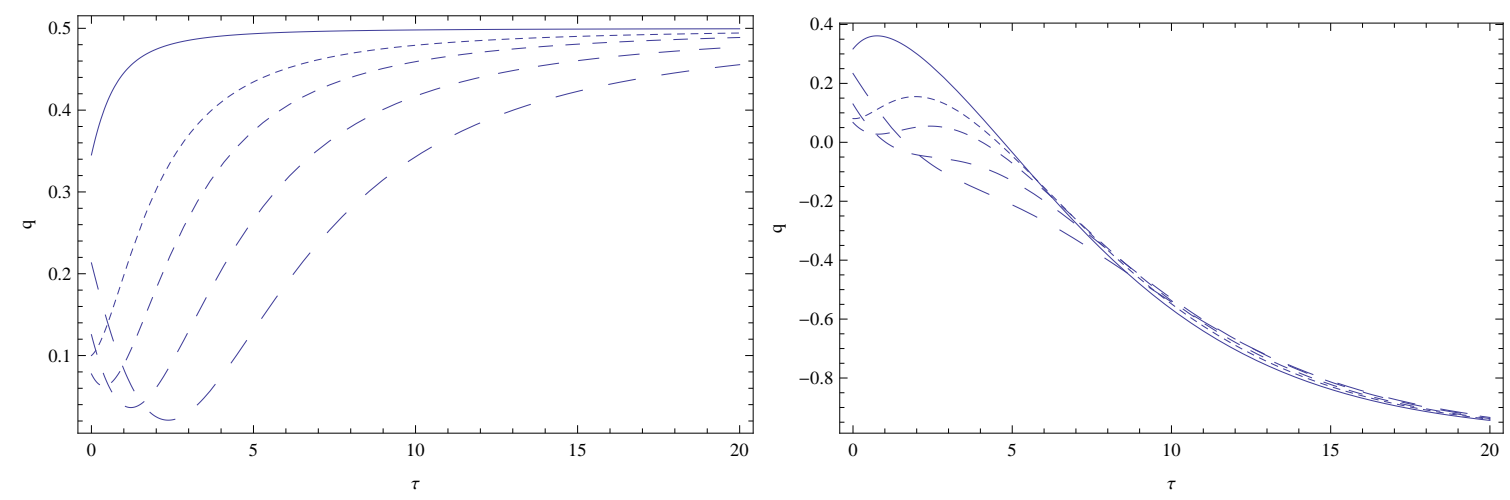

Figure 4. Time evolution of the deceleration parameter $q$ of the dust-matter Universe, as a function of $\tau$, for $\lambda=0$ (left panel) and for $\lambda=0.004$ (right panel), for different values of $k: k=1$ (solid curve), $k=3$ (dotted curve), $k=5$ (short dashed curve), $k=9$ (dashed curve) and $k=16$ (long dashed curve). The initial value of the energy density is $\theta(0)=0.20$.

We close this subsection by mentioning that more complex behaviors of $w_{D E}$ can also be achieved. In particular, according to (2.22), if during the cosmological expansion for some $\rho=\rho_{c r}$ the condition

$$
\frac{1}{3}(2 q-1)\left(\frac{\rho_{c r}}{\rho_{D E}}+1\right)=w_{D E}^{(c r)}=-1
$$

is satisfied, then at the corresponding time the phantom divide crossing will be realized.

\subsection{The radiation dominated phase}

Let us now investigate the cosmological behavior of a radiation dominated Universe, with the matter pressure satisfying the equation of state $p=\rho / 3$. The gravitational field equations (2.11), (2.12) and (2.18) give

$$
\ddot{\rho}=\frac{60 \beta_{4} \rho \dot{\rho}^{2}+64 \beta_{4} \rho^{4}+48 \Lambda \rho^{2}-9 \dot{\rho}^{2}+48 \rho^{3}}{48 \beta_{4} \rho^{2}},
$$



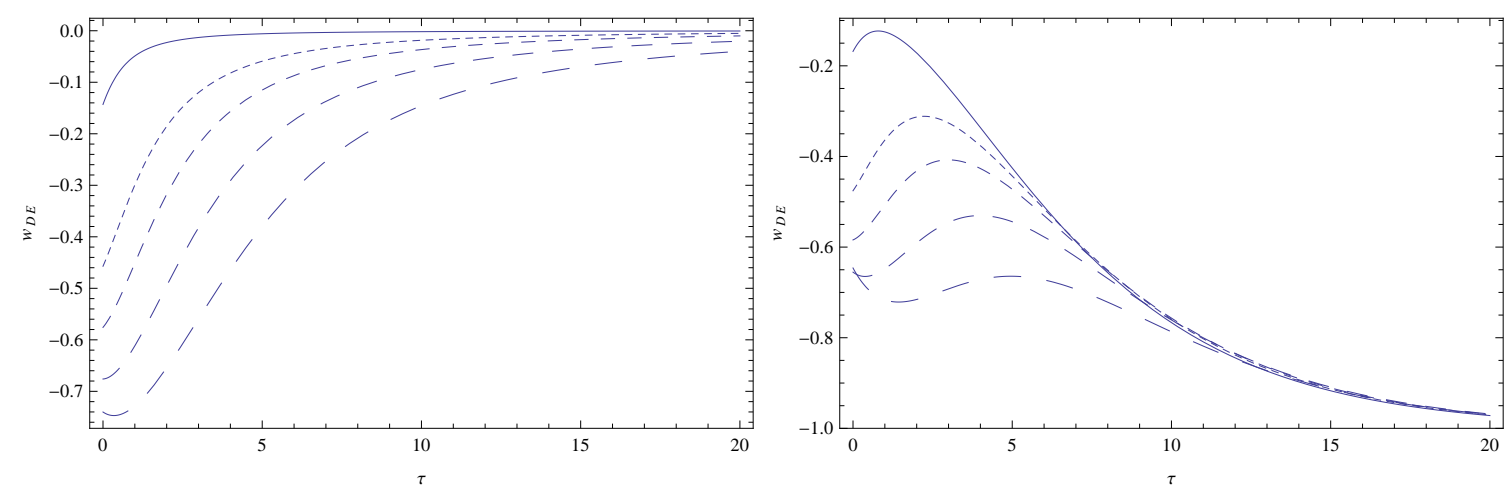

Figure 5. Time evolution of the dark-energy equation-of-state parameter $w_{D E}$ of the dust-matter Universe, as a function of $\tau$, for $\lambda=0$ (left panel) and for $\lambda=0.004$ (right panel), for different values of $k: k=1$ (solid curve), $k=3$ (dotted curve), $k=5$ (short dashed curve), $k=9$ (dashed curve) and $k=16$ (long dashed curve). The initial value of the energy density is $\theta(0)=0.20$.

$$
\ddot{\rho}=\frac{30 \beta_{4} \rho \dot{\rho}^{2}+32 \beta_{4} \rho^{4}+9 \dot{\rho}^{2}+24 \rho^{3}}{3 \rho\left(8 \beta_{4} \rho+3\right)}
$$

and

$$
\dot{\rho}+4 H \rho=0,
$$

respectively. It is interesting to note that for the radiation-dominated Universe of this subsection the dynamics is determined solely by the parameter $\beta_{4}$, with the parameter $\beta_{1}$ eliminated from the equations. The consistency condition requiring the equality of the right-hand-sides of equations (3.8) and (3.9) provides the basic evolution equation describing the dynamical behavior, namely

$$
\dot{\rho}=-4 \rho \sqrt{\frac{3 \Lambda+\left(3+8 \beta_{4} \Lambda\right) \rho+4 \beta_{4} \rho^{2}}{3\left(4 \beta_{4} \rho+3\right)}} .
$$

In the case $\Lambda=0$ and $\beta_{4}=0$, we obtain

$$
\dot{\rho}=-\frac{4 \rho^{3 / 2}}{\sqrt{3}}
$$

which yields the general solution

$$
\rho=\frac{3 \rho_{0}}{\left(2 \sqrt{\rho_{0}} t+\sqrt{3}\right)^{2}},
$$

where we have used the initial condition $\rho(0)=\rho_{0}$. Therefore, in this case we re-obtain the cosmological behavior of the radiation filled Universes in standard cosmology, with

$$
\begin{aligned}
& H(t)=\frac{\sqrt{\rho_{0}}}{2 \sqrt{\rho_{0}} t+\sqrt{3}}, \\
& a(t)=a_{0} \sqrt{2 t \sqrt{\frac{\rho_{0}}{3}}+1,}
\end{aligned}
$$

and $q=1$, respectively. 


\subsection{Stiff fluid cosmology}

One of the most common equations of state of high energy density cosmological matter, which has been used extensively to study the properties of the early Universe, is the linear barotropic equation of state, given by $p=(\gamma-1) \rho$, with $\gamma=$ constant $\in[1,2]$. A very important subcase is its so-called causal limit, corresponding to $\gamma=2$, which gives the Zeldovich, or stiff-fluid equation of state $p=\rho$ [81]. The Zeldovich equation of state applies to densities significantly higher than nuclear densities, $\rho>10 \rho_{\text {nuc }}$, with $\rho_{\text {nuc }}=10^{14} \mathrm{~g} / \mathrm{cm}^{3}$. From a field theoretical point of view the Zeldovich equation of state can be obtained by constructing a relativistic Lagrangian that allows bare nucleons to interact attractively via scalar meson exchange and repulsively via the exchange of a more massive vector meson [81]. On the other hand, in the non-relativistic limit both the quantum and classical field theories yield Yukawa-type potentials. At the highest densities the vector-meson exchange dominates, and by using a mean field approximation one can show that in the extreme limit of infinite densities the pressure tends to the energy density, namely $p \rightarrow \rho$ [81]. In this limit the sound speed $c_{s}^{2}=d p / d \rho \rightarrow 1$, and hence this equation of state satisfies the causality condition, with the speed of sound less than the speed of light.

For a stiff fluid the field equations (2.11), (2.12) and (2.18) provide respectively

$$
\begin{gathered}
\ddot{\rho}=\frac{12 \beta_{1} \rho \dot{\rho}^{2}-36 \beta_{1} \rho^{4}+18 \beta_{4} \rho \dot{\rho}^{2}+36 \beta_{4} \rho^{4}-\dot{\rho}^{2}+12 \rho^{3}}{12 \beta_{4} \rho^{2}}, \\
\ddot{\rho}=\frac{-\beta_{1} \rho \dot{\rho}^{2}+12 \beta_{1} \rho^{4}-5 \beta_{4} \rho \dot{\rho}^{2}-12 \beta_{4} \rho^{4}-\dot{\rho}^{2}-6 \rho^{3}}{\rho\left(6 \beta_{1} \rho-2 \beta_{4} \rho-1\right)},
\end{gathered}
$$

and

$$
\dot{\rho}+6 H \rho=0 .
$$

We mention that we have set to zero the explicit cosmological constant (namely $\Lambda=0$ ), since it is negligible comparing to the high densities that are needed to justify the Zeldovich stiff equation of state.

The basic evolution equation that describes the energy density evolution, obtained by equating the right hand sides of (3.16) and (3.17), is given by

$$
\dot{\rho}=-2 \sqrt{3} \sqrt{\frac{\rho^{3}\left[6\left(\beta_{1}-\beta_{4}\right)\left(3 \beta_{1}+\beta_{4}\right) \rho^{2}-\left(9 \beta_{1}+\beta_{4}\right) \rho+1\right]}{2 \rho\left[-9 \beta_{1}-2 \beta_{4}+12\left(\beta_{1}+\beta_{4}\right)\left(3 \beta_{1}+\beta_{4}\right) \rho\right]+1}} .
$$

By introducing the set of dimensionless quantities $(\tau, \theta, k, h)$, defined as

$$
\tau=\frac{2 \sqrt{3}}{\beta_{4}^{1 / 2}} t, \quad \theta=\beta_{4} \rho, \quad k=\frac{\beta_{1}}{\beta_{4}}, \quad h=\frac{\beta_{4}^{1 / 2}}{2 \sqrt{3}} H,
$$

equation (3.19) takes the final form

$$
\dot{\theta}=-\theta^{3 / 2} \sqrt{\frac{\theta[6(k-1)(3 k+1) \theta-9 k-1]+1}{2 \theta[12(k+1)(3 k+1) \theta-9 k-2]+1}} .
$$




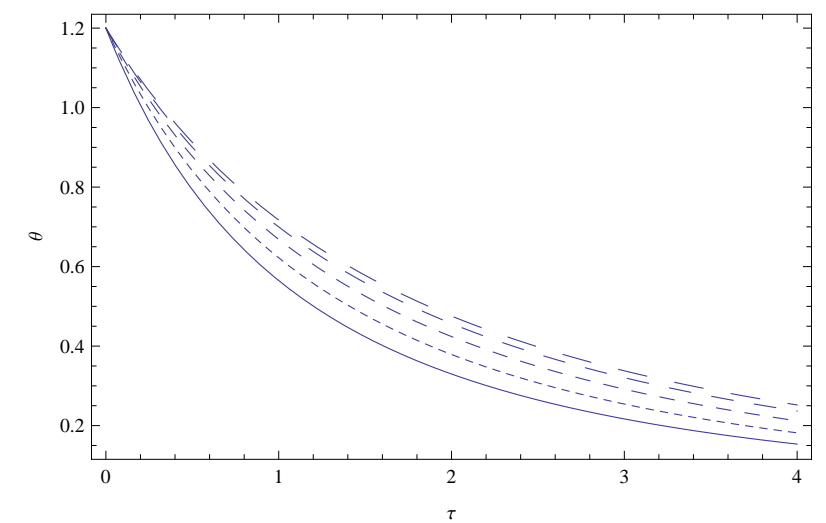

Figure 6. Time evolution of the matter energy density $\theta$ of the stiff-fluid Universe as a function of $\tau$, for different values of $k: k=-1$ (solid curve), $k=-3$ (dotted curve), $k=-5$ (short dashed curve), $k=-9$ (dashed curve) and $k=-16$ (long dashed curve). The initial value of the energy density is $\theta(0)=1.20$.

Thus, using (3.18) the Hubble function becomes

$$
h=\frac{1}{6} \sqrt{\frac{\theta[\theta(6(k-1)(3 k+1) \theta-9 k-1)+1]}{2 \theta[12(k+1)(3 k+1) \theta-9 k-2]+1}},
$$

which leads to a scale factor of the form

$$
a=a_{0} \theta^{-1 / 6} .
$$

Additionally, using (2.20) the deceleration parameter becomes

$$
q=2+\frac{3[(9 k+1) \theta-2]}{\theta[6(k-1)(3 k+1) \theta-9 k-1]+1}+\frac{6-6(9 k+2) \theta}{2 \theta[12(k+1)(3 k+1) \theta-9 k-2]+1} .
$$

We numerically evolve the scenario at hand for various parameter values, and in Figs. 68 we respectively depict the time evolution of the matter energy density, of the scale factor, of the scaled Hubble function, of the deceleration parameter and of the dark-energy equation-of-state parameter.

As we observe, for the considered numerical values of the model parameters the cosmological evolution of the Universe is strongly decelerating for all times, with the numerical values of the deceleration parameter in the range $1.2<q<2.2$. This is an expected result, since we have neglected the presence of the cosmological constant $\Lambda$ in the field equations. In physical terms it is also expected, since from the cosmological point of view the stiff-fluid regime lasts for a very short period during the evolution of the early Universe, in which the Zeldovich density conditions are satisfied. During this period the Universe is expanding at a slow rate, with the Hubble function monotonically decreasing in time. Furthermore, the dark-energy equation-of-state parameter has small negative values, being very close to zero for all considered dimensionless times.

At this point, let us remind that in standard General Relativity the evolution of the stiff-fluid Universe is given by $\rho_{G R}=\rho_{0} / a^{6}, \dot{H}_{G R}=-\rho_{G R}$, and $q_{G R}=2$. Hence, we 

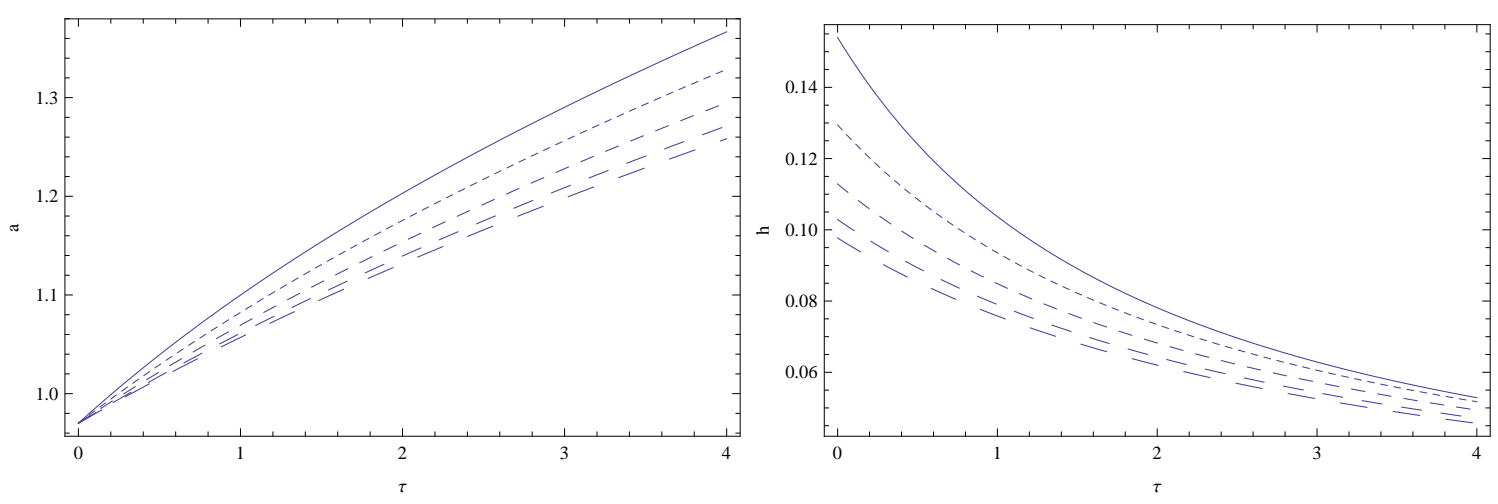

Figure 7. Time evolution of the scale factor a (left panel) and of the Hubble function $h$ (right panel) of the stiff-fluid Universe as a function of $\tau$, for different values of $k: k=-1$ (solid curve), $k=-3$ (dotted curve), $k=-5$ (short dashed curve), $k=-9$ (dashed curve) and $k=-16$ (long dashed curve). The initial value of the energy density is $\theta(0)=1.20$.
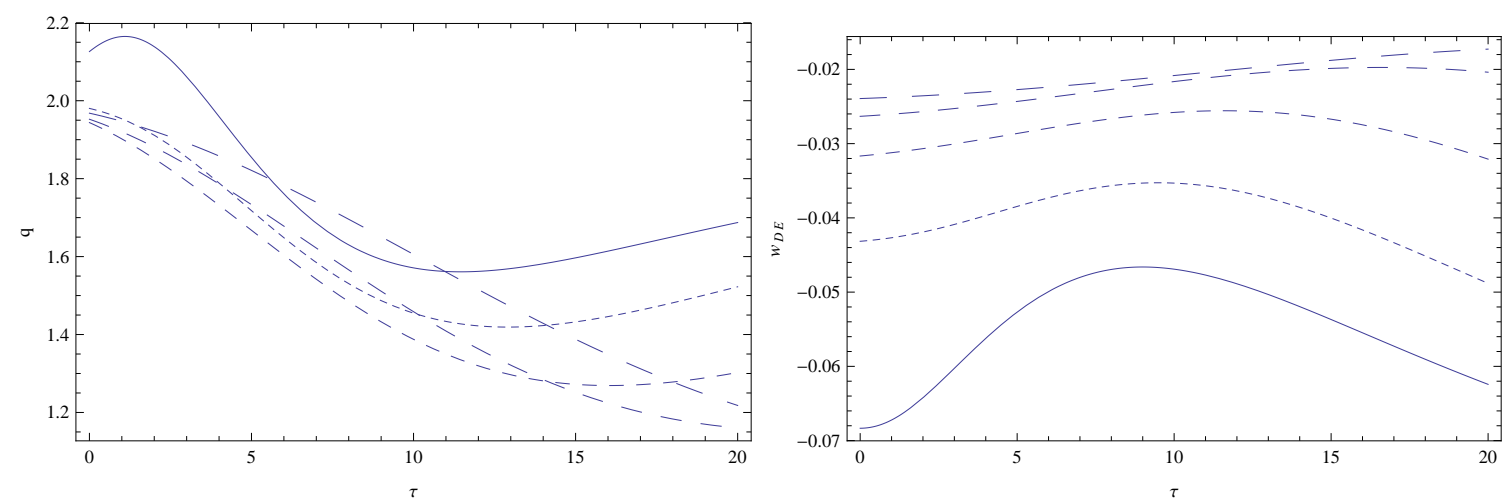

Figure 8. Time evolution of the deceleration parameter $q$ (left panel) and of the dark-energy equation of state parameter $w_{D E}$ (right panel) of the stiff-fluid Universe as a function of $\tau$, for different values of $k: k=-1$ (solid curve), $k=-3$ (dotted curve), $k=-5$ (short dashed curve), $k=-9$ (dashed curve) and $k=-16$ (long dashed curve). The initial value of the energy density is $\theta(0)=1.20$.

deduce that the presence of the higher derivative matter terms in the gravitational field equations leads to a significant departure from the standard cosmological dynamics. This feature could lead to strong nucleosynthesis constraints on the present scenario, and used to distinguish it from other modified gravity classes.

\section{Conclusions}

In the present work we have investigated the cosmological implications of a new class of modified gravity, which arises from the introduction of non-dynamical auxiliary fields. This feature leads the equations of motion to contain higher order derivatives of the matter fields. Although this could place tight observational constraints on this theory, as long as these constraints are satisfied the above class corresponds to a novel modified gravitational 
theory and cosmology that is worthy to explore. In particular, extracting the gravitational field equations and imposing a flat, homogeneous and isotropic geometry, we obtained the Friedmann equations, in which the effective dark-energy sector contains higher derivatives of the matter energy density and pressure.

One important feature of the present scenario is the conservation of the matter energymomentum tensor, which imposes strong constraints on the cosmological evolution. In particular, due to this conservation the density - scale-factor relation is the same as in standard General Relativity, which in the case of a barotropic cosmological matter fluid with $p=(\gamma-1) \rho$ takes the form $\rho \propto a^{-3 \gamma}$. However, since the evolution of the scale factor is now different, the matter-density time evolution differs from the standard General Relativistic one.

In the case where the matter sector is dust-like $(p=0)$, the Universe approaches asymptotically the de Sitter stage with $H \approx H_{0}=$ constant and with the matter content decreasing according to $\rho \approx \rho_{0} \exp \left(-3 H_{0} t\right)$, where both the deceleration parameter $q$ and the dark-energy equation-of-state parameter $w_{D E}$ tend to -1 , due to the domination of the explicit cosmological constant. This result is independent of the model parameters as expected, since in all cases the decrease of the matter energy density due to the expansion leads all the terms in the matter-dependent effective dark-energy sector to disappear, apart from the cosmological constant one. Hence, in the presence of the cosmological constant the de Sitter stage is an attractor solution of the field equations. This was actually expected, since as we stated in subsection 2.1, in the case of vanishing energy-momentum tensor the new non-GR terms vanish too, which is indeed the case in the above asymptotic regime. We stress however that at intermediate times the scenario at hand can be very different than GR. Finally, on the other hand, in the absence of an explicit cosmological constant, the Universe results in a non-accelerating, matter-dominated Universe.

In the case of radiation matter $p=\rho / 3$, and in the absence of an explicit cosmological constant we obtained a non-accelerating Universe, similar to the radiation-dominated phase of standard General Relativity. Additionally, in the case of a stiff matter $p=\rho$, which is expected to be realized in the very early Universe, we found that the Universe is expanding at a slow rate, with the effective dark-energy equation-of-state parameter having small negative values.

The above variety of cosmological evolutions reveals that the theory with higher matter derivatives is indeed new, and deserves further investigation. More specifically, it would be interesting and necessary to perform a detailed cosmological perturbation analysis, in order to examine the stability properties of the theory, as well as to confront it with perturbationrelated observables such as the large-scale structure and the growth index. Moreover, one could perform a full phase space analysis, which will reveal the global, asymptotic behavior of the scenario. Furthermore, another important avenue of analysis would be to use data from Type Ia Supernovae (SNIa), Baryon Acoustic Oscillations (BAO), and Cosmic Microwave Background (CMB) observations, in order to impose constraints on the theory. The above investigations may also provide specific signatures and effects, which could distinguish between this theory and other alternatives of modified gravity. We aim to explore in detail these issues in upcoming publications. 


\section{Appendix}

\section{A Divergence-free character of the tensor $S_{a b}$}

In this Appendix, we will deduce the conditions imposed in order for the tensor $S_{a b}$ to be divergence-free, namely $\nabla_{a} S^{a b}=0$. Indeed, the tensor $S_{a b}$ possesses the following properties: i) it is imposed to vanish in vacuum, $T_{a b}=0$; ii) it is divergence-free, due to the Bianchi identity, $\nabla_{a} G^{a b}=0$, and the fact that $T_{a b}$ is also divergence-free, namely $\nabla_{a} T^{a b}=0$. Note that the latter property, in this theory, arises due to the fact that matter is minimally coupled to the metric, which guarantees $\nabla_{a} T^{a b}=0$. Thus, these theories, that possess auxiliary fields, correspond to modifying the Einstein field equation by adding a divergence-free tensor that vanishes in vacuum and that depends on the metric, the energy-momentum tensor and its derivatives.

Now, it is important to emphasize that the form of $S_{a b}$, considered in this work and in Ref. [69], is given as an expansion in orders of the matter fields $T$ and their derivatives, namely equation (2.1) is given in terms of a derivative expansion. Hence, it is by definition approximate and not exact. One cannot expect to get exact vanishing of the divergence precisely for this reason. Instead, what is important is that order by order in the expansion $\nabla_{a} S^{a b}$ is zero to that order. For instance, one maintains terms which are $O\left(T^{2}\right)$, while terms such as $\Lambda \beta_{1}^{2} \nabla^{b}\left(T^{2}\right)$ contribute to higher orders. Thus, if one assigns two derivatives to $T$ then one essentially keeps up to four derivatives. More specifically, consider $k$ a parameter that counts derivatives in this way, then indeed one has $\nabla_{a} S^{a b}=0+O\left(k^{6}\right)$ which is all that is needed, as $O\left(k^{6}\right)$ terms were not included anyway in the original expansion (note that this is a standard procedure in any perturbation theory).

In this context, the tensor $S_{a b}$, up to fourth order in the derivatives, is thus given by equation (2.1), which we reproduce here

$$
\begin{aligned}
S_{a b}= & \alpha_{1} g_{a b} T+\alpha_{2} g_{a b} T^{2}+\alpha_{3} T T_{a b}+\alpha_{4} g_{a b} T_{c d} T^{c d}+\alpha_{5} T^{c}{ }_{a} T_{c b} \\
& +\beta_{1} \nabla_{a} \nabla_{b} T+\beta_{2} g_{a b} \square T+\beta_{3} \square T_{a b}+2 \beta_{4} \nabla^{c} \nabla_{(a} T_{b) c}+\ldots
\end{aligned}
$$

In order to impose that $S_{a b}$ is divergence-free, to the required order, the following relations are imposed:

$$
\begin{aligned}
\left(\square \nabla_{b}-\nabla_{b} \square\right) T & =R_{a b} \nabla^{a} T, \\
\left(\nabla^{a} \nabla^{c} \nabla_{a}-\nabla^{c} \square\right) T_{c b} & =R_{a b c d} \nabla^{d} T^{c a}, \\
\nabla^{a} R_{a b c d} & =2 \nabla_{[c} R_{d] b},
\end{aligned}
$$

and taking into account the lowest-order equation given by $R_{a b}=T_{a b}-\alpha g_{a b} T+g_{a b} \Lambda+\ldots$ (once again, we refer the reader to Ref. [69] for more details), lead to the necessary cancellations between terms to $O\left(k^{6}\right)$. Note, however, that equations (A.2)-(A.4) are exact identities. 
Finally, the resulting modified Einstein equation takes the form of equation (2.3), which we reproduce here

$$
\begin{aligned}
G_{a b}= & T_{a b}-\Lambda g_{a b}-\beta_{1} \Lambda g_{a b} T+\frac{1}{4}\left(1-2 \beta_{1} \Lambda\right)\left(\beta_{1}-\beta_{4}\right) g_{a b} T^{2} \\
& +\left[\beta_{4}\left(1-2 \beta_{1} \Lambda\right)-\beta_{1}\right] T T_{a b}+\frac{1}{2} \beta_{4} g_{a b} T_{c d} T^{c d}-2 \beta_{4} T^{c}{ }_{a} T_{c b} \\
& +\beta_{1} \nabla_{a} \nabla_{b} T-\beta_{1} g_{a b} \square T-\beta_{4} \square T_{a b}+2 \beta_{4} \nabla^{c} \nabla_{(a} T_{b) c}+\ldots
\end{aligned}
$$

\section{Acknowledgments}

The authors are grateful to Paolo Pani and Thomas Sotiriou for helpful clarifications and comments.FSNL acknowledges financial support of the Fundação para a Ciência e Tecnologia through an Investigador FCT Research contract, with reference IF/00859/2012, funded by FCT/MCTES (Portugal), and grants CERN/FP/123618/2011 and EXPL/FISAST $/ 1608 / 2013$. The research of ENS is implemented within the framework of the Operational Program "Education and Lifelong Learning" (Actions Beneficiary: General Secretariat for Research and Technology), and is co-financedby the European Social Fund (ESF) and the Greek State.

\section{References}

[1] E. J. Copeland, M. Sami and S. Tsujikawa, Dynamics of dark energy, Int. J. Mod. Phys. D 15, 1753 (2006), [arXiv:hep-th/0603057].

[2] B. Ratra and P. J. E. Peebles, Cosmological Consequences of a Rolling Homogeneous Scalar Field, Phys. Rev. D 37, 3406 (1988).

[3] C. Wetterich, Cosmology and the Fate of Dilatation Symmetry, Nucl. Phys. B 302, 668 (1988).

[4] A. R. Liddle and R. J. Scherrer, A Classification of scalar field potentials with cosmological scaling solutions, Phys. Rev. D 59, 023509 (1999), [arXiv:astro-ph/9809272].

[5] Z. -K. Guo, N. Ohta and Y. -Z. Zhang, Parametrizations of the dark energy density and scalar potentials, Mod. Phys. Lett. A 22, 883 (2007), [arXiv:astro-ph/0603109].

[6] S. Dutta, E. N. Saridakis and R. J. Scherrer, Dark energy from a quintessence (phantom) field rolling near potential minimum (maximum), Phys. Rev. D 79, 103005 (2009), [arXiv: 0903.3412].

[7] T. Harko, F. S. N. Lobo and M. K. Mak, Arbitrary scalar field and quintessence cosmological models, Eur. Phys. J. C 74, 2784 (2014), [arXiv: 1310.7167].

[8] R. R. Caldwell, A Phantom menace?, Phys. Lett. B 545, 23 (2002), [arXiv:astro-ph/9908168].

[9] R. R. Caldwell, M. Kamionkowski and N. N. Weinberg, Phantom energy and cosmic doomsday, Phys. Rev. Lett. 91, 071301 (2003), [arXiv:astro-ph/0302506].

[10] S. 'i. Nojiri and S. D. Odintsov, Quantum de Sitter cosmology and phantom matter, Phys. Lett. B 562, 147 (2003), [arXiv: hep-th/0303117]. 
[11] V. K. Onemli and R. P. Woodard, Quantum effects can render $w<-1$ on cosmological scales, Phys. Rev. D 70, 107301 (2004), [arXiv:gr-qc/0406098].

[12] E. N. Saridakis, Theoretical Limits on the Equation-of-State Parameter of Phantom Cosmology, Phys. Lett. B 676, 7 (2009), [arXiv: 0811.1333].

[13] Y. F. Cai, E. N. Saridakis, M. R. Setare and J. Q. Xia, Quintom Cosmology: Theoretical implications and observations, Phys. Rept. 493, 1 (2010), [arXiv:0909.2776].

[14] Z. K. Guo, Y. S. Piao, X. M. Zhang and Y. Z. Zhang, Cosmological evolution of a quintom model of dark energy, Phys. Lett. B 608, 177 (2005), [arXiv:astro-ph/0410654].

[15] M. R. Setare and E. N. Saridakis, Coupled oscillators as models of quintom dark energy, Phys. Lett. B 668, 177 (2008), [arXiv: 0802. 2595].

[16] C. Armendariz-Picon, V. F. Mukhanov and P. J. Steinhardt, Essentials of k essence, Phys. Rev. D 63, 103510 (2001), [arXiv: astro-ph/0006373].

[17] L. P. Chimento, Extended tachyon field, Chaplygin gas and solvable $k$-essence cosmologies, Phys. Rev. D 69, 123517 (2004), [arXiv:astro-ph/0311613].

[18] A. Nicolis, R. Rattazzi and E. Trincherini, The Galileon as a local modification of gravity, Phys. Rev. D 79, 064036 (2009), [arXiv: 0811.2197].

[19] S. Capozziello and M. De Laurentis, Extended Theories of Gravity, Phys. Rept. 509, 167 (2011), [arXiv: 1108.6266].

[20] S. Capozziello, Curvature quintessence, Int. J. Mod. Phys. D 11, 483 (2002), [arXiv: gr-qc/0201033].

[21] T. Chiba, 1/R gravity and scalar - tensor gravity, Phys. Lett. B 575, 1 (2003), [arXiv: astro-ph/0307338].

[22] G. Allemandi, A. Borowiec, M. Francaviglia and S. D. Odintsov, Dark energy dominance and cosmic acceleration in first order formalism, Phys. Rev. D 72, 063505 (2005), [arXiv: gr-qc/0504057].

[23] S. 'i. Nojiri and S. D. Odintsov, Modified $f(R)$ gravity consistent with realistic cosmology: From matter dominated epoch to dark energy universe, Phys. Rev. D 74, 086005 (2006), [arXiv:hep-th/0608008].

[24] S. 'i. Nojiri and S. D. Odintsov, Unifying inflation with LambdaCDM epoch in modified $f(R)$ gravity consistent with Solar System tests, Phys. Lett. B 657, 238 (2007), [arXiv:0707.1941].

[25] L. Amendola, R. Gannouji, D. Polarski and S. Tsujikawa, Conditions for the cosmological viability of $f(R)$ dark energy models, Phys. Rev. D 75, 083504 (2007), [arXiv: gr-qc/0612180].

[26] A. A. Starobinsky, Disappearing cosmological constant in $f(R)$ gravity, JETP Lett. 86, 157 (2007), [arXiv:0706.2041].

[27] N. Lanahan-Tremblay and V. Faraoni, The Cauchy problem of $f(R)$ gravity, Class. Quant. Grav. 24, 5667 (2007), [arXiv:0709.4414].

[28] C. G. Boehmer, T. Harko and F. S. N. Lobo, Dark matter as a geometric effect in $f(R)$ gravity, Astropart. Phys. 29, 386 (2008), [arXiv:0709.0046].

[29] T. Harko, T. S. Koivisto, F. S. N. Lobo and G. J. Olmo, Metric-Palatini gravity unifying 
local constraints and late-time cosmic acceleration, Phys. Rev. D 85, 084016 (2012), [arXiv: 1110.1049].

[30] G. R. Bengochea and R. Ferraro, Dark torsion as the cosmic speed-up, Phys. Rev. D 79, 124019 (2009), [arXiv:0812.1205].

[31] E. V. Linder, Einstein's Other Gravity and the Acceleration of the Universe, Phys. Rev. D 81, 127301 (2010) [Erratum-ibid. D 82, 109902 (2010)], [arXiv: 1005.3039].

[32] S. H. Chen, J. B. Dent, S. Dutta and E. N. Saridakis, Cosmological perturbations in $f(T)$ gravity, Phys. Rev. D 83, 023508 (2011), [arXiv: 1008.1250].

[33] L. Iorio and E. N. Saridakis, Solar system constraints on $f(T)$ gravity, Mon. Not. Roy. Astron. Soc. 427, 1555 (2012), [arXiv: 1203.5781].

[34] Z. Haghani, T. Harko, H. R. Sepangi, and S. Shahidi, Weyl-Cartan-Weitzenböck gravity as a generalization of teleparallel gravity, JCAP 10, 061 (2012), [arXiv:1202.1879].

[35] Z. Haghani, T. Harko, H. R. Sepangi, and S. Shahidi, Weyl-Cartan-Weitzenböck gravity through Lagrange multiplier, Phys. Rev. D 88, 044024 (2013), [arXiv:1307.2229].

[36] S. 'i. Nojiri, S. D. Odintsov and M. Sasaki, Gauss-Bonnet dark energy, Phys. Rev. D 71, 123509 (2005), [arXiv:hep-th/0504052].

[37] T. Koivisto and D. F. Mota, Cosmology and Astrophysical Constraints of Gauss-Bonnet Dark Energy, Phys. Lett. B 644, 104 (2007), [arXiv:astro-ph/0606078].

[38] M. De Laurentis, Topological invariant quintessence, Mod. Phys. Lett. A 30, no. 12, 1550069 (2015) [arXiv: 1411.7001].

[39] P. Horava, Quantum Gravity at a Lifshitz Point, Phys. Rev. D 79, 084008 (2009), [arXiv: 0901.3775$]$.

[40] E. Kiritsis and G. Kofinas, Horava-Lifshitz Cosmology, Nucl. Phys. B 821, 467 (2009), [arXiv: 0904.1334].

[41] E. N. Saridakis, Horava-Lifshitz Dark Energy, Eur. Phys. J. C 67, 229 (2010), [arXiv:0905.3532].

[42] C. Bogdanos and E. N. Saridakis, Perturbative instabilities in Horava gravity, Class. Quant. Grav. 27, 075005 (2010), [arXiv:0907.1636].

[43] E. N. Saridakis, Constraining Horava-Lifshitz gravity from neutrino speed experiments, Gen. Rel. Grav. 45, 387 (2013), [arXiv:1110.0697].

[44] C. de Rham and G. Gabadadze, Generalization of the Fierz-Pauli Action, Phys. Rev. D 82, 044020 (2010), [arXiv: 1007.0443].

[45] K. Hinterbichler, Theoretical Aspects of Massive Gravity, Rev. Mod. Phys. 84, 671 (2012), [arXiv:1105.3735].

[46] Y. -F. Cai, C. Gao and E. N. Saridakis, Bounce and cyclic cosmology in extended nonlinear massive gravity, JCAP 1210 (2012) 048, [arXiv:1207.3786].

[47] C. de Rham, Massive Gravity, [arXiv:1401.4173].

[48] K. S. Stelle, Renormalization of Higher Derivative Quantum Gravity, Phys. Rev. D 16, 953 (1977).

[49] J. -P. Uzan, Cosmological scaling solutions of nonminimally coupled scalar fields, Phys. Rev. D 59, 123510 (1999), [arXiv: gr-qc/9903004]. 
[50] L. Amendola, Scaling solutions in general nonminimal coupling theories, Phys. Rev. D 60, 043501 (1999), [arXiv:astro-ph/9904120].

[51] Y. Fujii and K. Maeda, The scalar-tensor theory of gravitation, Cambridge, USA: Univ. Pr. (2003).

[52] A. De Felice and S. Tsujikawa, Generalized Galileon cosmology, Phys. Rev. D 84, 124029 (2011), [arXiv: 1008.4236].

[53] C. Deffayet, X. Gao, D. A. Steer and G. Zahariade, From k-essence to generalised Galileons, Phys. Rev. D 84, 064039 (2011), [arXiv:1103.3260].

[54] A. De Felice and S. Tsujikawa, Conditions for the cosmological viability of the most general scalar-tensor theories and their applications to extended Galileon dark energy models, JCAP 1202, 007 (2012), [arXiv:1110.3878].

[55] G. Leon and E. N. Saridakis, Dynamical analysis of generalized Galileon cosmology, JCAP 1303, 025 (2013), [arXiv: 1211.3088].

[56] O. Bertolami, C. G. Boehmer, T. Harko and F. S. N. Lobo, Extra force in $f(R)$ modified theories of gravity, Phys. Rev. D 75, 104016 (2007), [arXiv:0704.1733].

[57] O. Bertolami, F. S. N. Lobo and J. Paramos, Non-minimum coupling of perfect fluids to curvature, Phys. Rev. D 78, 064036 (2008), [arXiv:0806.4434].

[58] O. Bertolami, J. Paramos, T. Harko and F. S. N. Lobo, Non-minimal curvature-matter couplings in modified gravity, arXiv:0811.2876 [gr-qc];

[59] O. Bertolami and J. Paramos, Mimicking dark matter through a non-minimal gravitational coupling with matter, JCAP 1003, 009 (2010), [arXiv:0906.4757].

[60] T. Harko, Modified gravity with arbitrary coupling between matter and geometry, Phys. Lett. B 669, 376 (2008), [arXiv:0810.0742].

[61] T. Harko and F. S. N. Lobo, $f\left(R, L_{m}\right)$ gravity, Eur. Phys. J. C 70, 373 (2010), [arXiv: 1008.4193].

[62] T. Harko, F. S. N. Lobo and O. Minazzoli, Extended $f\left(R, L_{m}\right)$ gravity with generalized scalar field and kinetic term dependences, Phys. Rev. D 87, no. 4, 047501 (2013), [arXiv: 1210.4218].

[63] J. Wang and K. Liao, Energy conditions in $f(R, L(m))$ gravity, Class. Quant. Grav. 29, 215016 (2012), [arXiv: 1212.4656].

[64] T. Harko, F. S. N. Lobo, S. 'i. Nojiri and S. D. Odintsov, $f(R, T)$ gravity, Phys. Rev. D 84, 024020 (2011), [arXiv:1104.2669].

[65] Z. Haghani, T. Harko, F. S. N. Lobo, H. R. Sepangi and S. Shahidi, "Further matters in space-time geometry: $f\left(R, T, R_{\mu \nu} T^{\mu \nu}\right)$ gravity, Phys. Rev. D 88, no. 4, 044023 (2013), [arXiv: 1304.5957].

[66] S. D. Odintsov and D. Sez-Gmez, $f\left(R, T, R_{\mu \nu} T^{\mu \nu}\right)$ gravity phenomenology and $\Lambda C D M$ universe, Phys. Lett. B 725, 437 (2013), [arXiv: 1304.5411].

[67] T. Harko, F. S. N. Lobo, G. Otalora and E. N. Saridakis, Non-minimal torsion-matter coupling extension of $f(T)$ gravity, Phys. Rev. D 89, 124036 (2014), [arXiv:1404.6212].

[68] T. Harko, F. S. N. Lobo, G. Otalora and E. N. Saridakis, $f(T, \mathcal{T})$ gravity and cosmology, JCAP 12, 021 (2014), [arXiv: 1405.0519]. 
[69] P. Pani, T. P. Sotiriou and D. Vernieri, Gravity with Auxiliary Fields, Phys. Rev. D 88, 121502 (2013), [arXiv: 1306.1835].

[70] M. Baados and D. Cohen, A short note on gravity with tensor auxiliary fields, [arXiv: 1309.6177].

[71] B. Guo, Y. -X. Liu and K. Yang, Brane worlds in gravity with auxiliary fields, [arXiv: 1405.0074].

[72] S. Capozziello, J. Matsumoto, S. Nojiri and S. D. Odintsov, Dark energy from modified gravity with Lagrange multipliers, Phys. Lett. B 693, 198 (2010), [arXiv:1004.3691].

[73] Y. -F. Cai and E. N. Saridakis, Cyclic cosmology from Lagrange-multiplier modified gravity, Class. Quant. Grav. 28, 035010 (2011), [arXiv: 1007.3204].

[74] Y. -F. Cai and E. N. Saridakis, Non-singular Cyclic Cosmology without Phantom Menace, J. Cosmol. 17, 7238 (2011), [arXiv: 1108.6052].

[75] J. J. Rawal and J. V. Narlikar, On a nonlinear and Lorentz Invariant version of Newtonian gravitation, Jour. Astrophys. Astron. 3, 393 (1982).

[76] J. V. Narlikar and T. Padmanabhan, On a nonlinear and Lorentz Invariant version of Newtonian gravitation - II, Jour. Astrophys. Astron. 6, 171 (1985).

[77] N. Tamanini and T. S. Koivisto, Consistency of nonminimally coupled $f(R)$ gravity, Phys. Rev. D 88, 064052 (2013) [arXiv: 1308.3401].

[78] I. Ayuso, J. B. Jimenez and A. de la Cruz-Dombriz, On the consistency of universally non-minimally coupled $f\left(R, T, R_{\mu \nu} T^{\mu \nu}\right)$ theories, [arXiv:1411.1636].

[79] T. Padmanabhan, Cosmological constant: The Weight of the vacuum, Phys. Rept. 380, 235 (2003), [arXiv:hep-th/0212290].

[80] M. Sami and T. Padmanabhan, A Viable cosmology with a scalar field coupled to the trace of the stress tensor, Phys. Rev. D 67, 083509 (2003) [Erratum-ibid. D 67, 109901 (2003)], [arXiv:hep-th/0212317].

[81] S. L. Shapiro and S. A. Teukolsky, Black holes, white dwarfs and neutron stars, New York, John Wiley \& Sons, (1983). 